EPJ Web of Conferences 45, 01055 (2013)

DOI: $10.1051 /$ epjconf/20134501055

(C) Owned by the authors, published by EDP Sciences, 2013

\title{
LDA measurements and turbulence spectral analysis in an agitated vessel
}

\author{
Bohuš Kysela ${ }^{\mathrm{a}}$, Jiří Konfršt, and Zdeněk Chára \\ Institute of Hydrodynamics, AS CR, v.v.i. \\ B. Kysela, J. Konfršt, and Z. Chára
}

\begin{abstract}
During the last years considerable improvement of the derivation of turbulence power spectrum from Laser Doppler Anemometry (LDA) has been achieved. The irregularly sampled LDA data is proposed to approximate by several methods e.g. Lomb-Scargle method, which estimates amplitude and phase of spectral lines from missing data, methods based on the reconstruction of the auto-correlation function (referred to as correlation slotting technique), methods based on the reconstruction of the time series using interpolation between the uneven sampling and subsequent resampling etc. These different methods were used on the LDA data measured in an agitated vessel and the results of the power spectrum calculations were compared. The measurements were performed in the mixing vessel with flat bottom. The vessel was equipped with four baffles and agitated with a six-blade pitched blade impeller. Three values of the impeller speed (Reynolds number) were tested. Long time series of the axial velocity component were measured in selected points. In each point the time series were analyzed and evaluated in a form of power spectrum.
\end{abstract}

\section{Introduction}

The mixing operation is commonly used phenomenon in several industries namely chemical and process engineering. Agitated vessels have an important role in these areas in long therm. The design of a big industrial agitated vessels is developed through modeling of the particular processes in small-scale conditions. The results are then transferred to lager scope by the scale-up modeling. In that case most processes are designed and controlled through the values of the power input per unit mass, therefore the turbulence and the dissipation rate seems to have an essential role in that modeling, at least for high Reynolds number. For the flow field measurements LDA (Laser Doppler Anemometry) was used and the time ensemble averaged mean velocities were obtained, moreover, the time series of the local velocities enabled the analysis of turbulence spectra as well as correlation functions.Subsequently the turbulence spectra and correlation functions can be used to derive time and length scales and dissipation rate. The direct estimation of the spatial correlations is possible only using multi-point measurements. For single-point measurements the temporal correlation function have to be transformed to spatial functions using the Taylor's frozen turbulence hypothesis.

$$
\begin{gathered}
\kappa=\frac{\omega}{\bar{u}}=\frac{2 \pi f}{\bar{u}}, \\
r=\bar{u} t,
\end{gathered}
$$

where the $\kappa$ is the wave number, $\omega=2 \pi f$ circular frequency, $r$ spatial distance, $t$ time and $\bar{u}$ mean velocity respectively. The relation between the power spectrum $G(f)$ and the wave number spectrum $E(\kappa)$ is following:

$$
E(\kappa)=E\left(\frac{2 \pi f}{\bar{u}}\right)=\frac{\bar{u}}{2 \pi} G(f),
$$

and spatial and temporal auto-correlation function relation:

$$
R_{r}(r)=R_{t}\left(\frac{r}{\bar{u}}\right)
$$

The LDA data is unevenly sampled so the spectral analysis requires nonstandard processing. The estimation of spectral analysis from LDA data was reviewed by Benedict at al. [1] and classified them to the following groups:

- a direct transform;

- reconstruction with equidistant re-sampling and Fourier transform.

- a slotting technique and a cosine transform;

One possibility is to use as a direct transform the LombScargle method instead of common Fourier transform to performed spectral analysis on unevenly sampled data described e.g. in [2]. Several modifications of signal reconstuction methods published Simon at al. [3], Broersen [4], Moreau at al. [5] and recently Plantier at al. [6]. The slotting technique used Tummers \& Passchier [7], Nobach [8] who also used and improved the reconstruction method and established the web page: http://ldvproc.nambis.de for LDA signal and data processing, which contains also program source codes.

\subsection{Lomb-Scargle method}

The velocity data set $u_{i}$ for $i=1,2, \ldots, N$ at observation times $t_{i}$ for Lomb-Scargle perodiogram, the mean and variance are computed first:

$$
\bar{u}=\frac{1}{N} \sum_{1}^{n} u_{i}, \quad \sigma^{2}=\frac{1}{N-1} \sum_{1}^{N}\left(u_{i}-\bar{u}\right),
$$

\footnotetext{
a e-mail: kysela@ih.cas.cz
} 
then compute a time offset $\tau$ for each angular frequency $\omega=2 \pi f>0$ by

$$
\tan (2 \omega \tau)=\frac{\sum_{j} \sin 2 \omega t_{j}}{\sum_{j} \cos 2 \omega t_{j}} .
$$

The perodiogram of the spectral power as a function of the angular frequency is

$$
\begin{aligned}
P_{N}(\omega)=\frac{1}{2 \sigma^{2}}\left\{\frac{\left[\sum_{j}\left(u_{j}-\bar{u}\right) \cos \omega\left(t_{j}-\tau\right)\right]^{2}}{\sum_{j} \cos ^{2} \omega\left(t_{j}-\tau\right)}\right. \\
\left.+\frac{\left[\sum_{j}\left(u_{j}-\bar{u}\right) \sin \omega\left(t_{j}-\tau\right)\right]^{2}}{\sum_{j} \sin ^{2} \omega\left(t_{j}-\tau\right)}\right\} .
\end{aligned}
$$

A principle of fast evaluation is described by Press \& Rybicki [2].

\subsection{Reconstruction method}

The reconstruction method is based on the reconstruction of velocity signal from LDA samples and their equidistant resampling. Then the power spectrum could be estimated by Fourier transform as well as the auto-correlation function via the inverse Fourier transform. The simple reconstruction is obtained by the Sample and Hold reconstruction:

$$
u^{\prime}(t)=u_{i} \quad \text { for } t_{i} \leqslant<t_{i+1}, \quad i=1, \ldots, N,
$$

and then the equidistant resampling with steps $\Delta \tau$ :

$$
u_{i}^{\prime}=u^{\prime}(i \Delta \tau) i \quad \text { for } i=1, \ldots, N_{R}-1,
$$

the obtained equidistant data series can be readily processed by Fourier transform, moreover, if the block $N_{R}$ is power of 2. It is useful to use the fast Fourier transform (FFT) algorithm. The Fourier transform is

$$
U_{j}^{\prime}=\sum_{i=1}^{N_{R}-1} u_{i}^{\prime} e^{-2 \pi i j \mathbb{I} / N_{R}} \quad \text { for } j=1, \ldots, N_{R}-1,
$$

and the power spectral density is

$$
\hat{S}_{j}^{\prime}=\frac{\Delta \tau}{N_{R}}\left|U_{j}^{\prime}\right|^{2} \quad \text { for } j=1, \ldots, N_{R}-1
$$

The used method is very well described in [1].

\subsection{Slot correlation}

The slot correlation was used at first to estimate the autocorrelation function (ACF) from which the power spectral density could be calculated by the Fourier transform. The auto-correlation function is obtained from

$$
\begin{gathered}
\hat{R}_{k}=\hat{R}(k \Delta \tau)=\frac{\hat{\sigma}_{u}^{2} A}{\sqrt{B C}}, \\
A=\sum_{i=1}^{N-1} \sum_{j=i+1}^{N} u_{i} u_{j} w_{i} w_{j} b_{k}\left(t_{j}-t_{i}\right),
\end{gathered}
$$

$$
\begin{aligned}
& B=\sum_{i=1}^{N-1} \sum_{j=i+1}^{N} u_{i}^{2} w_{i} w_{j} b_{k}\left(t_{j}-t_{i}\right), \\
& C=\sum_{i=1}^{N-1} \sum_{j=i+1}^{N} u_{j}^{2} w_{i} w_{j} b_{k}\left(t_{j}-t_{i}\right),
\end{aligned}
$$

where the $u_{i}$ and $u_{j}$ are two samples taken at times $t_{i}$ and $t_{j}, w_{i}$ and $w_{j}$ are the weighting factors (e.g. transit time $w_{i}=t_{T T ; i}$, and $b_{k}(\Delta t)$ is the fuzzy mask function:

$$
b_{k}\left(t_{j}-t_{i}\right)=\left\{\begin{array}{ll}
1-\left|\frac{\Delta t}{\Delta \tau}-k\right| & \text { for }\left|\frac{\Delta t}{\Delta \tau}-k\right|<1 \\
0 & \text { otherwise }
\end{array},\right.
$$

and the velocity variance:

$$
\hat{\sigma}_{u}^{2}=\frac{\sum_{i=1}^{N} u_{i}^{2} w_{i}}{\sum_{i=1}^{N} w_{i}} .
$$

The autocorrelation function transform to the power spectral density is

$$
\hat{S}(f)=\Delta \tau \sum_{k=-K}^{K-1} \hat{R}_{k} w_{k} \cos (2 \pi f k \Delta \tau),
$$

where the frequency dependent variable windowing is implemented e.g. Tuckey-Hannig window:

$$
w_{k}=\left\{\begin{array}{ll}
\frac{1}{2}-\frac{1}{2} \cos \left(\frac{\pi k f \Delta \tau}{c_{6}}\right) & \text { for }|k f \Delta \tau|<c_{6} \\
0 & \text { otherwise }
\end{array} .\right.
$$

The parameter $c_{6}$ can be chosen arbitrarily e.g. $c_{6}=6$ was found to give good results. The slot correlation method is closely described in [8].

\subsection{Data blocks averaging}

The error can be reduced by smoothing the estimated spectrum. The smoothing could by implemented by splitting raw data into blocks of equal duration. Each block is an independent auto-correlation function and power spectrum. The final auto-correlation function $\langle\bar{R}\rangle$ and power spectrum $\langle\bar{S}\rangle$ are determined as the average of separate blocks.

$$
\begin{aligned}
& \langle\bar{R}\rangle=\frac{1}{N_{b}} \sum_{i=1}^{N_{B}} \hat{R}^{(i)}, \\
& \langle\bar{S}\rangle=\frac{1}{N_{b}} \sum_{i=1}^{N_{B}} \hat{S}^{(i)},
\end{aligned}
$$

where the upper index ( $i$ ) represents the $i-$ th block. The number and length of blocs is proportional to the resolution of power spectrum so the settings chosen will be a compromise between resolution and variance.

\section{Experimental in an agitated vessel}

Experiments were carried out in a pilot plant flat bottomed cylindrical vessel with diameter $T=300 \mathrm{~mm}$. The vessel was provided with four radial baffles at its wall. The baffles width was $B / T=1 / 10$. A liquid aspect ratio in agitated 


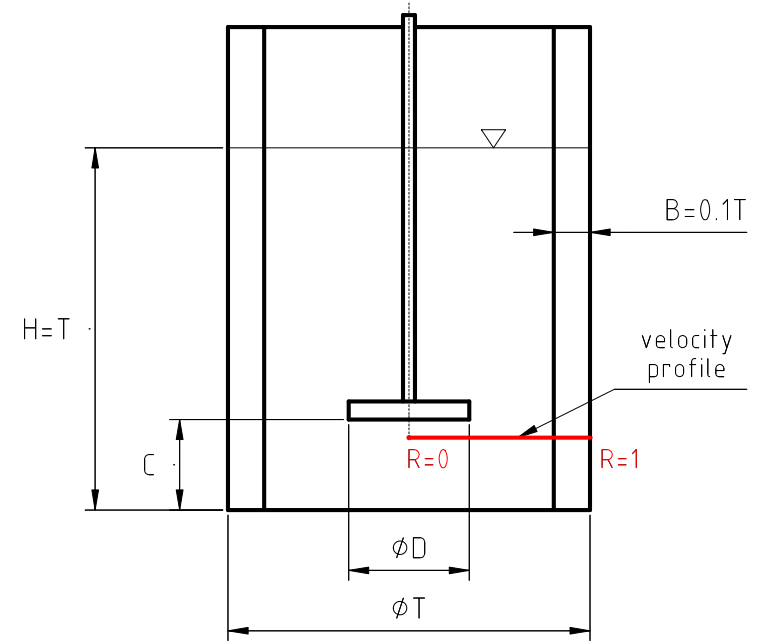

Fig. 1. Agitated vessel and impeller setup.

Table 1. Conditions of experiments related to the impeller speed.

\begin{tabular}{lll}
\hline $\begin{array}{l}\text { Impeller } \\
\text { speed }[\mathrm{rpm}]\end{array}$ & $\begin{array}{l}\mathrm{Re} \\
{[-]}\end{array}$ & $\begin{array}{l}\bar{\epsilon} \\
{\left[\mathrm{m}^{2} \mathrm{~s}^{-3}\right]}\end{array}$ \\
\hline 300 & 49900 & 0.094 \\
450 & 74850 & 0.398 \\
600 & 99800 & 0.755 \\
\hline
\end{tabular}

vessel was $H / T=1$ (see figure 1). Distilled water at room temperature (density $\rho=998 \mathrm{~kg} \cdot \mathrm{m}^{-3}$, dynamic viscosity $\mu=1 \mathrm{mPa} \cdot \mathrm{s}$ ) was used as agitated liquid. The impeller was six-pitch blade turbine CVS 691020 with diameter $D=$ $100 \mathrm{~mm}(T / D=3)$. The flow regimes in an agitated vessel are related to the Reynolds number which is defined for agitated vessel:

$$
\operatorname{Re}=\frac{n D^{2}}{v}
$$

where $D$ is the impeller diameter, $n$ impeller speed and $v$ kinematic viscosity. The power consumption to agitated volume could be calculated from:

$$
P=P_{o} \rho n^{3} D^{5}
$$

where $\rho$ is mixed liquid density, $P_{o}$ is power number which becomes constant in region with fully developed turbulence $R e>10^{4}$. It means that the power number is independent of the Reynolds number and the value of the dissipated energy is only dependent of the mixing vessel geometry (power number for six-pitch blade turbine is $\left.P_{o}=1.6\right)$. The mean energy dissipation per unit mass can be derivated:

$$
\bar{\epsilon}=\frac{P}{\rho V}=\frac{P_{o} n^{3} D^{5}}{V},
$$

where $V$ is the agitated volume. The dissipated energy dependency in fully turbulent region can be expressed for a similar geometry in the form

$$
\bar{\epsilon}=C_{g} n^{3} D^{2}
$$

where $C_{g}$ is constant dependent of the geometry.

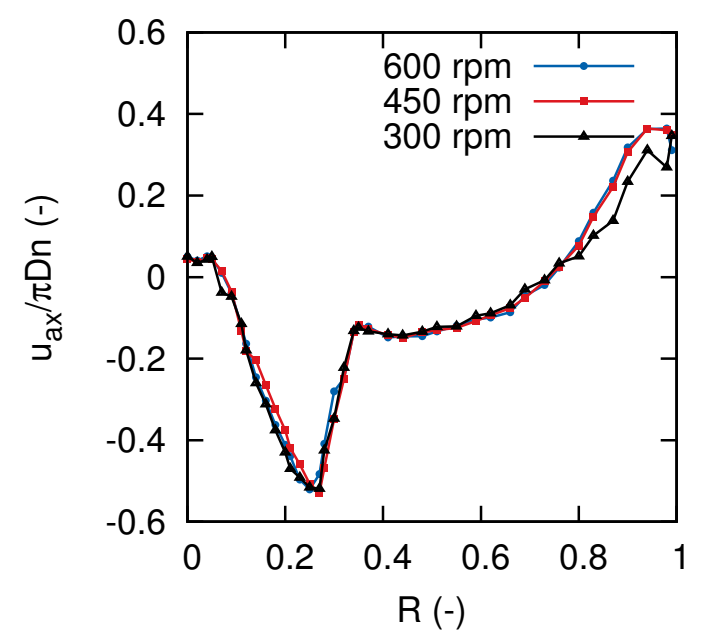

Fig. 2. Dimensionless profiles of ensemble-averaged mean axial velocity normalized by the impeller peripheral velocity.

\subsection{LDA velocity measurements}

The LDA measurements of axial velocity in agitated vessel were performed. The series of point measurements by using the automatic traversing support were compiled to the axial velocity profile, the setup is described in [9]. The velocity profile was measured $15 \mathrm{~mm}$ under the impeller and from impeller axis $(\mathrm{R}=0)$ to the vessel wall $(\mathrm{R}=1)$ see figure 1 . The profiles for the three impeller speed conditions were measured: 300; 450; $600 \mathrm{rpm}$. The obtained ensemble-averaged mean velocity profiles are depicted in figure 2. The root mean square values of the fluctuations are shown in figure 3 . The depicted results of velocities are presented in dimensionless form where the velocities are divided by an impeller peripheral velocity.

One component LDA system set-up consists of: CASIX LDC 1500 laser supply with power $100 \mathrm{~mW}$ and wavelength $532 \mathrm{~nm}$, DANTEC 57x transmitting optics, DANTEC receiving optics with photomultiplier 57x08 and P80 DANTEC BSA processor. The set-up was supervised by BSA FLOW SOFTWARE v3.0 installed on standard PC where the data was processed. S-HGS (Silver coated - Hollow Glass Spheres) with mean diameter $10 \mu m$ and density $1.1 \mathrm{~g} \cdot \mathrm{cm}^{-3}$ were used as trace particles.

\subsection{Evaluation of power spectra}

The methods for power spectra estimations where described in section 1 . Two points in the measured profile were chosen for the power spectra analysis. The first one $(R=0.59)$ is in the secondary stream produced by the impeller and the second one $(R=0.20)$ was in the main stream from the impeller. The time dependency of velocity in second point is known to be strongly anisotropic and is affected by trailing vortices. It means that it contains the frequencies related to the impeller speed and the number of the impeller blades. This fact was used to recognize the validity of the power spectra estimation methods. The mean data rate frequency in each measured point was around $1 \mathrm{kHz}$ and the acquired 


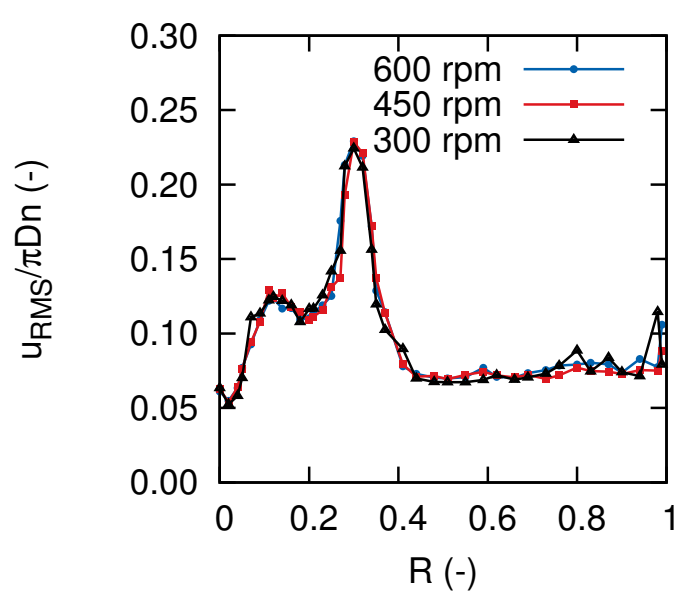

Fig. 3. Dimensionless profiles of root mean square deviation of the mean axial velocity normalized by the impeller peripheral velocity.

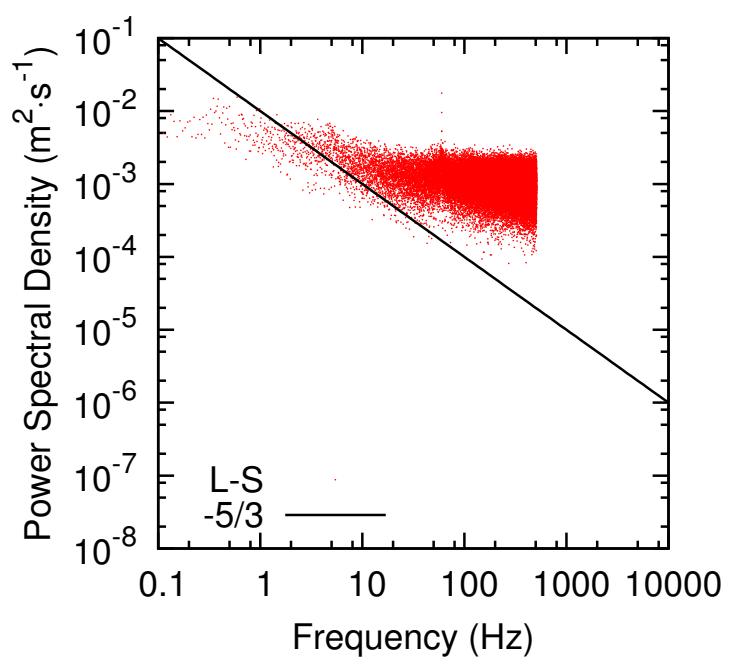

Fig. 4. Power spectrum for $600 \mathrm{rpm}, R=0.20$ and full range 500000 data points: Lomb-Scargle(L-S), law $-5 / 3$.

data points were around $500000(500 s)$ which was exactly dependent on the measurement conditions namely velocity.

The first was used the Lomb-Scargle method described in section 1.1. The result of power spectra with full range data (500 000 points) is depicted in figure 4 . The bias in higher frequencies is obvious. It is in agreement with Benedict at al. investigation [1].

Two other methods were compared: Slot correlation (SC) see section 1.3 and Sample and hold reconstruction $(\mathrm{S}+\mathrm{H})$ see section 1.2. In figure 5 are depicted the results of the comparison from full data range 500000 points. Both methods show three peaks at the frequency spectrum. The most significant peak can be observed at frequency $60 \mathrm{~Hz}$, which corresponds to the impeller speed $10 s^{-1}(600 \mathrm{rpm})$ multiplied by 6 (number of blades). The peak occurring at frequency $120 \mathrm{~Hz}$ is the second-harmonic one. The peak at frequency $6 \mathrm{~Hz}$ is proportional to the oscillations of the primary circulation loop [10]. The results of frequencies

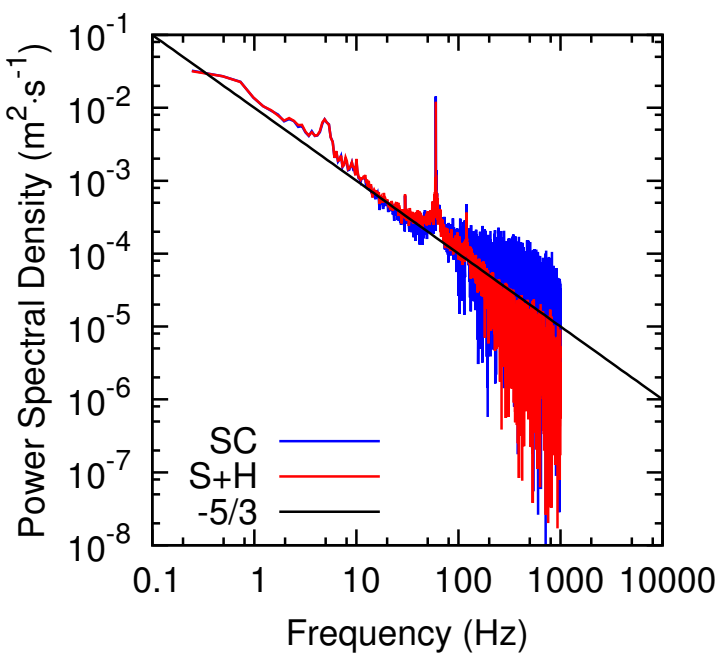

Fig. 5. Power spectrum for $600 \mathrm{rpm}, R=0.20$ and full range 500000 data points: Slot correlation (SC); Sample and hold reconstruction $(\mathrm{S}+\mathrm{H})$; law $-5 / 3$.

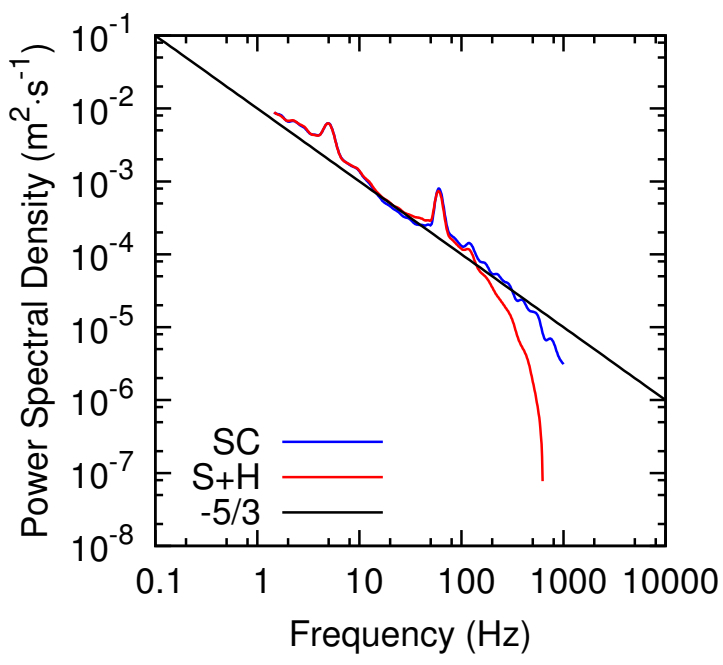

Fig. 6. Power spectrum for $600 \mathrm{rpm}, R=0.20$ variable windowing with size 8192 data points: Slot correlation (SC); Sample and hold reconstruction $(\mathrm{S}+\mathrm{H})$; law $-5 / 3$.

above $100 \mathrm{~Hz}$ oscillate and the block method is subsequently used to remove this phenomena. The smooth results are depicted in figure 6 .

The next step was to compare the results of the three impeller speeds. Compared results of the slot correlation method are shown in figure 7 . All the three observed peaks obviously show the dependency on the impeller speed. The deformation in the spectrum from $450 \mathrm{rpm}$ above $120 \mathrm{~Hz}$ is probably affected by lower data rate.

The frequency power spectra were transformed to wave number spectra using the Taylor hypothesis (see section 1.). The spectra for both examined points $R=0.20$ and $R=0.59$ are shown in figure 8 and 9 respectively. In point $R=0.59$ the slope of spectra deviates from Kolmogorof theoretical turbulent slope $(-5 / 3)$, nevertheless the spectra of all three measurements indicate identical tendency. 


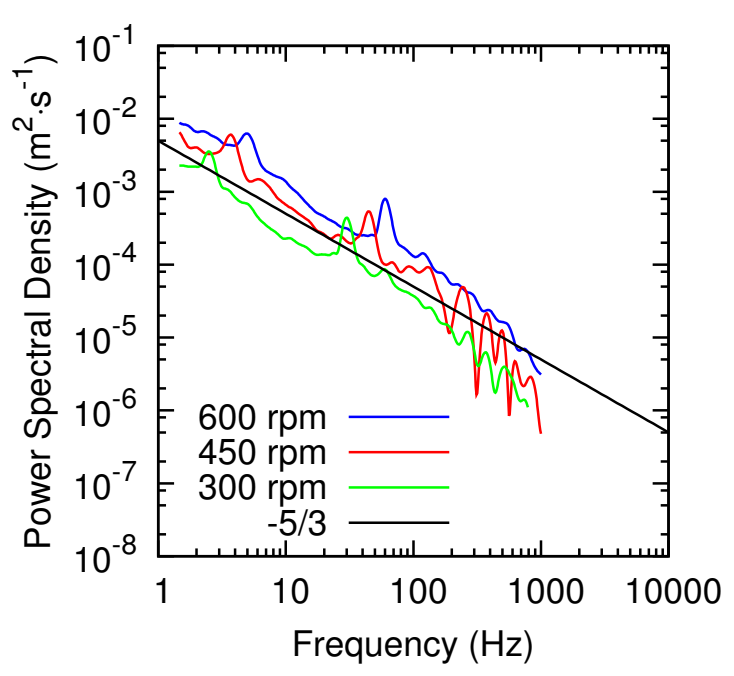

Fig. 7. Comparison of power spectra obtained by (SC) in point $R=0.20: 600 \mathrm{rpm} ; 450 \mathrm{rpm} ; 300 \mathrm{rpm}$; law $-5 / 3$.

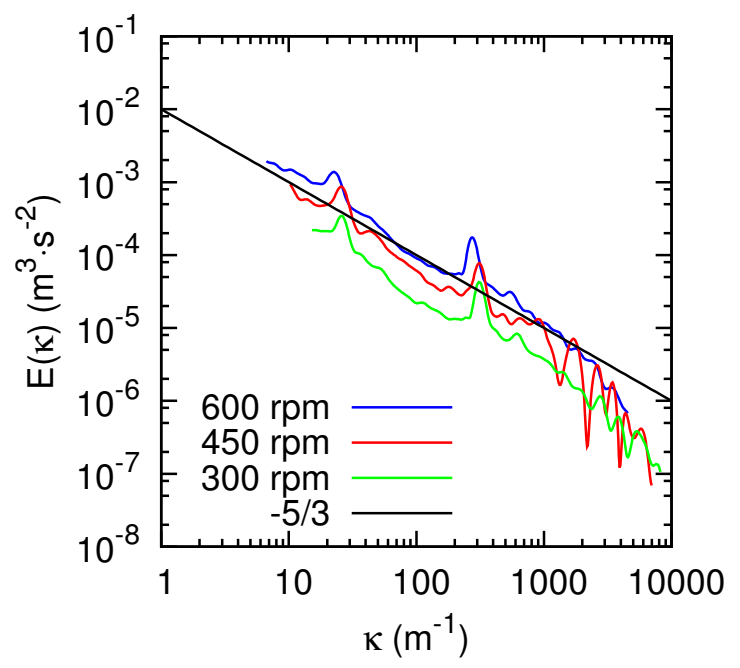

Fig. 8. Comparison of wave number spectra in point $R=0.20$ : $600 \mathrm{rpm} ; 450 \mathrm{rpm} ; 300 \mathrm{rpm}$; law $-5 / 3$.

\section{Length scales and dissipation rate}

The obtained temporal correlation function and power spectra were transformed by using Taylor hypothesis described in section 1. to spatial correlation and wave number spectra (figures 8-9). The spatial correlation can be used to derive the integral length scale and consequently dissipation energy [11].

Integral length scale can be obtained from:

$$
L=\frac{1}{\left\langle u^{\prime 2}\right\rangle} \int_{0}^{\infty} R_{r}(x) \mathrm{d} x,
$$

and the dissipation rate using turbulent kinetic energy can be derivated from

$$
\epsilon=\frac{k^{3 / 2}}{2 L}
$$

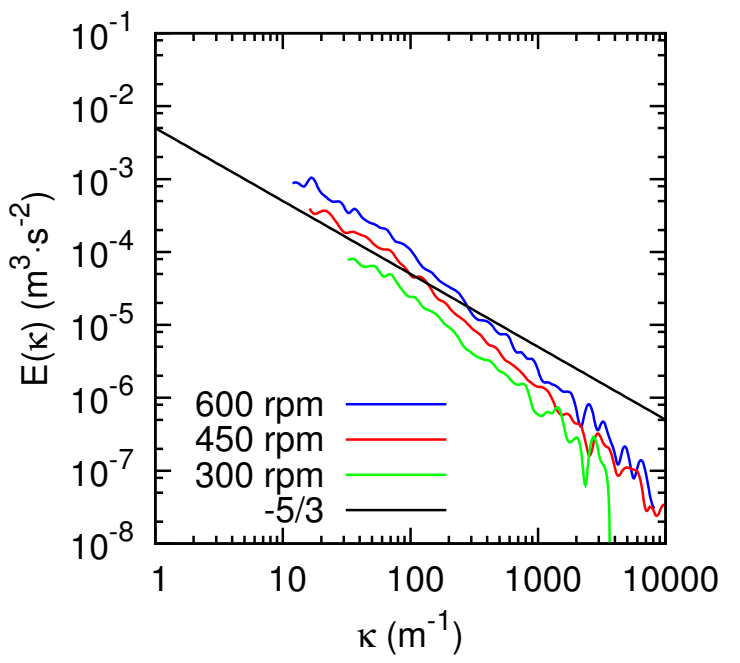

Fig. 9. Comparison of wave number spectra in point $R=0.59$ recalculated for wave number : $600 \mathrm{rpm} ; 450 \mathrm{rpm} ; 300 \mathrm{rpm}$; law $-5 / 3$.

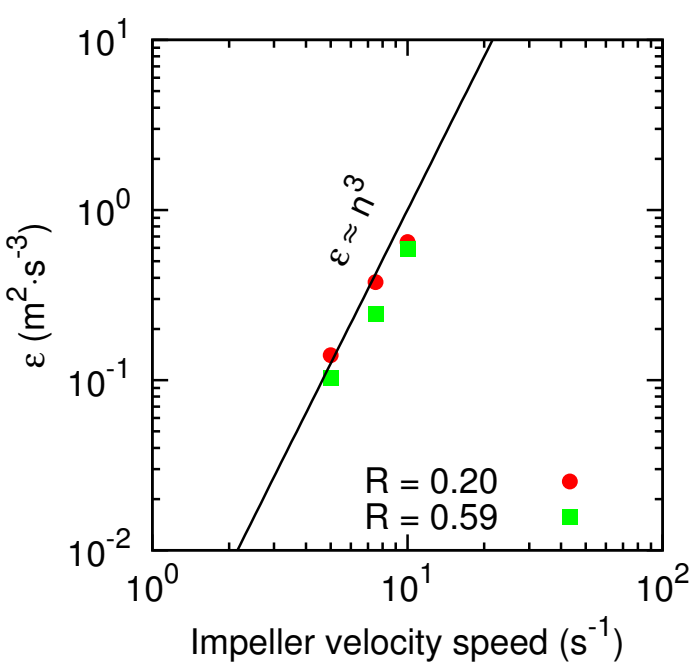

Fig. 10. The dependency of the dissipation energy vs. impeller velocity speed : results derived from integral length scale for $R=$ 0.20 (circles); results derived from integral length scale for $R=$ 0.59 (squares) table 2.

Table 2. The integral length scale and dissipation obtained from equations $26-27$.

\begin{tabular}{l|ll|ll}
\hline & $R=0.20$ & & $R=0.59$ & \\
\hline Impeller & $\mathrm{L}$ & $\epsilon$ & $\mathrm{L}$ & $\epsilon$ \\
speed $[\mathrm{rpm}]$ & {$[\mathrm{mm}]$} & {$\left[\mathrm{m}^{2} \mathrm{~s}^{-3}\right]$} & {$[\mathrm{mm}]$} & {$\left[\mathrm{m}^{2} \mathrm{~s}^{-3}\right]$} \\
\hline 300 & 40 & 0.14 & 10 & 0.10 \\
450 & 50 & 0.37 & 16 & 0.24 \\
600 & 61 & 0.44 & 15 & 0.59 \\
\hline
\end{tabular}

where $L$ is the integral length scale and $k$ is the turbulent kinetic energy, that can be obtained from:

$$
k=\frac{3}{2}\left\langle u^{\prime 2}\right\rangle \text {. }
$$




\section{Conclusions}

The velocity profiles of mean axial ensemble-averaged velocity were obtained by the one dimensional measurements performed by laser Doppler anemometry in an agitated vessel. In two selected points were used three methods to estimate the energy spectrum of turbulence: Lomb-Scargle, reconstruction and slot correlation. The slot correlation method seems to be more realistic than the two others in that case.

The comparisons of the turbulence energy spectrum for the three impeller speeds were performed. The power spectra in one point have identical shape for all three impeller speeds and the spectrum is shifted proportionally to the impeller speed.

The integral length scale and dissipation energy were derived and obtained from auto-correlation functions of the point data sets. The values of the integral length scale are in agreement with results of other autors [11]. The measurements of the three velocity components are necessary to attain better results as well as an improvement of the LDA setup to achieve a higher data rate in future.
2. W.H. Press, G.B. Rybicki, Astrophysical Journal 338, 277 (1989)

3. L. Simon, J. Fitzpatrick, Experiments in Fluids 37, 272 (2004), 10.1007/s00348-004-0814-2

4. P.M.T. Broersen, IEEE T. Instrumentation and Measurement 58, 1380 (2009)

5. S. Moreau, G. Plantier, J.C. Valiere, H. Bailliet, L. Simon, Experiments in Fluids 50, 179 (2011), 10.1007/s00348-010-0905-1

6. G. Plantier, S. Moreau, L. Simon, J.C. Valiere, A.L. Duff, H. Bailliet, Digital Signal Processing 22, 994 (2012)

7. M.J. Tummers, D.M. Passchier, Measurement Science and Technology 12, 1641 (2001)

8. H. Nobach, Experiments in Fluids 32, 337 (2002), 10.1007/s003480100362

9. I. Fort, B. Kysela, T. Jirout, Chemical and Process Engineering - Inzynieria Chemiczna i Procesowa 31, 661 (2010)

10. O. Bruha, T. Bruha, I. Fort, M. Jahoda, Acta Polytechnica 47, 17 (2007)

11. S.M. Kresta, P.E. Wood, Chemical Engineering Science 48, 1761 (1993)

\section{Symbols}

B baffle width, $\mathrm{m}$

C off-bottom clearance, $\mathrm{m}$

D impeller diameter, $\mathrm{m}$

f frequency, $\mathrm{s}^{-1}$

$\mathrm{H}$ total liquid depth, $\mathrm{m}$

$\mathrm{L}$ integral length scale, $\mathrm{m}$

$\mathrm{n}$ impeller speed, $\mathrm{s}^{-1}$

$\mathrm{P} \quad$ power required by the impeller, $\mathrm{W}$

$\mathrm{P}_{\mathrm{O}} \quad$ power number, -

$\mathrm{R}$ dimensionless radius of agitated vessel, -

Re Reynolds number, -

$\mathrm{T} \quad$ vessel diameter, $\mathrm{m}$

$\mathrm{u} \quad$ fluid velocity, $\mathrm{m} \cdot \mathrm{s}^{-1}$

$\mathrm{u}^{\prime} \quad$ fluctuation of velocity, $\mathrm{m} \cdot \mathrm{s}^{-1}$

$\mathrm{u}_{\mathrm{ax}} \quad$ mean axial velocity, $\mathrm{m} \cdot \mathrm{s}^{-1}$

$\mathrm{u}_{\mathrm{RMS}}$ root mean square of axial velocity, $\mathrm{m} \cdot \mathrm{s}^{-1}$

\section{Greek symbols}

$\epsilon$ rate of dissipation of kinetic energy of turbulence, $\mathrm{m}^{2} \cdot \mathrm{s}^{-3}$

$\bar{\epsilon} \quad$ mean energy dissipation per unit mass, $\mathrm{m}^{2} \cdot \mathrm{s}^{-3}$

$\kappa \quad$ wave number, $\mathrm{m}^{-1}$

$v$ kinematic viscosity of agitated liquid, $\mathrm{m}^{2} \cdot \mathrm{s}^{-1}$

$\rho$ density of agitated liquid, $\mathrm{kg} \cdot \mathrm{m}^{-3}$

\section{Acknowledgments}

This research has subsidized by the research project No.

GA ČR P101/12/2274 and RVO: 67985874.

\section{References}

1. L.H. Benedict, H. Nobach, C. Tropea, Measurement Science and Technology 11, 1089 (2000) 